\title{
Pokémon GO y su relación con la actividad física, orientación espacial y socialización en usuarios mexicanos \\ Pokémon GO and its relationship with physical activity, spatial orientation and socialization in mexican users
}

\author{
Argenis Peniel Vergara-Torres, José Luis Juvera-Portilla, Oswaldo Ceballos-Gurrola, Jorge Zamarripa \\ Universidad Autónoma de Nuevo León (México)
}

\begin{abstract}
Resumen. La práctica regular de actividad física es un predictor de importantes beneficios a la salud. En ese sentido, Pokémon GO es un juego de realidad aumentada para teléfonos móviles inteligentes en el que sus usuarios deben salir a caminar para dar cumplimiento a los objetivos del juego, lo que implica realización de actividad física. El objetivo de este estudio consistió en analizar la cantidad de tiempo de actividad física realizada, percepciones de mejora en habilidades de orientación espacial, socialización, conocimiento de nuevos sitios urbanos, así como en el medio natural y accidentes relacionados a jugar Pokémon GO en usuarios mexicanos. Los resultados mostraron diferencias significativas entre los días por semana y tiempo destinados a caminar antes y después de comenzar a jugar Pokémon GO, así como percepciones de mejora en habilidades de orientación espacial, socialización, asistencia a lugares de interés nuevos en la ciudad y en el medio natural, además de que los principales accidentes relacionados tienen que ver con elementos urbanos como postes, señales de tránsito o banquetas. En conclusión, a partir del uso de Pokémon GO, se ha identificado un incremento en la actividad física realizada, una percepción de mejora en la habilidad de orientación espacial, conocimiento de nuevas personas y visita de nuevos sitios de interés en la ciudad y en el medio natural, por lo que se puede revertir el tiempo de sedentarismo que podría ocasionar el uso del teléfono móvil, al jugar Pokémon GO.
\end{abstract}

Palabras clave: Videojuegos, realidad aumentada, Pokémon GO, actividad física, México.

\begin{abstract}
The regular practice of physical activity is a predictor of important health benefits. In that sense, Pokémon GO is an augmented reality game for smartphones in which users must walk to meet the objectives of the game, which involves physical activity. The objective of this study was to analyze the amount of physical activity performed, perceptions of improvement in spatial orientation skills, socialization, knowledge of new urban sites, as well as natural environment and accidents related to playing Pokémon GO in Mexican users. The results showed significant differences between days per week and time per day spent walking before and after starting to play Pokémon GO, as well as perceptions of improved spatial orientation skills, socialization, assistance to new places of interest in the city and in the natural environment in addition to the fact that the main related accidents refer to urban elements such as poles, traffic signs, or sidewalks. In conclusion, an increase in physical activity performed has been identified from the use of Pokémon $\mathrm{GO}$, as well as a perception of improvement in the ability of spatial orientation, knowledge of new people and visiting new places of interest in the city and the environment, so sedentary time, which is commonly encouraged by using of smartphone, could be reversed by playing Pokémon GO.
\end{abstract}

Keywords: Videogames, augmented reality, Pokémon, physical activity, Mexico.

\section{Introducción}

La inactividad física se ha asociado a una gran cantidad de problemas relacionados a la salud, entre los que se pueden destacar enfermedades cardiovasculares, el cáncer y la diabetes (Organización Mundial de la Salud [OMS], 2018). Los cambios en el estilo de vida de las personas ocasionados por la tecnología (Hallal, Wells, Reichert, Anselmi \& Victora, 2006), han propiciado el aumento del sedentarismo (López, Pozo, Fuentes \& Vicente, 2019), lo que se convierte en un problema mundial (OMS, 2018).

El desarrollo tecnológico en los teléfonos móviles, así como su uso, se ha incrementado notablemente durante los últimos años (Panova \& Carbonell, 2018). Se estima que, en México, 69.6 millones de personas utilizan teléfonos móviles inteligentes (smartphones) (Instituto Nacional de Estadística y Geografía [INEGI], 2018). Dichos dispositivos, a través de aplicaciones informáticas (apps), permiten al usuario la realización de una gran diversidad de tareas, ya sea de índole profesional, de educación, de acceso a servicios, así como de ocio y recreación (Aguilar-Martínez, Tort, Medina \& Saigí-

Fecha recepción: 04-02-20. Fecha de aceptación: 01-05-20

José Luis Juvera-Portilla

juveraportilla@gmail.com
Rubió, 2015; Lunde, Nilsson, Bergland, Kværner \& Bye, 2018; Wang, Xiang \& Fesenmaier, 2016).

Con relación a la actividad física, se han desarrollado numerosas apps que permiten administrar diversos aspectos de la actividad física (e.g., Google Fit, Pacer, Stepz, Pedometer, Nike+, entre otras), lo cual representa una importante herramienta que apoya y facilita la realización de la actividad física (Aznar, Cáceres, Trujillo \& Romero, 2019).

Por otra parte, pese a que el uso de videojuegos ha sido asociado a efectos negativos como la generación de adicción, problemas de atención y para relacionarse con los demás, conductas violentas, sedentarismo, además de problemas musculoesqueléticos como lesiones en tendones, dolor de manos, muñecas, espalda y cuello (Ayenigbara, 2018; Greitemeyer \& Mügge, 2014; Khang, Kim \& Kim, 2013; Klitsie et al., 2013; Moncada \& Chacón, 2012), también se han encontrado beneficios en su uso, como mejoras de habilidades cognitivas, oculo-manuales, la motivación y autoeficacia así como el incremento de los niveles de actividad física en el caso de los videojuegos activos (Buckley \& Anderson, 2006; Ferguson, 2007; Moncada \& Chacón, 2012; Peng, Crouse \& Lin, 2012). También conocidos como exergames, los videojuegos activos implican un formato en el que se combina actividad física y los elementos virtuales del juego (López, Suárez, Ruiz \& Martínez, 2017). Al respecto, diversas inves- 
tigaciones han encontrado que el uso de videojuegos activos, puede por sí mismo, conseguir o contribuir en alcanzar la cantidad y tipo de actividad física recomendada (Daley, 2009; Lozano-Sánchez et al., 2019; Staiano \& Calvert, 2011; Sween et al., 2014).

Los videojuegos también se han adaptado para su uso en teléfonos móviles y aunque la mayoría de ellos implica poca o nula actividad física, se han desarrollado algunos cuya finalidad es la realización de ésta (Aznar et al., 2019). En ese sentido, Pokémon $\mathrm{GO}^{\circledR}$ (Nianticlabs.com, 2019) es un videojuego de realidad aumentada disponible en teléfonos móviles, que permite a los jugadores encarnar el rol de entrenador pokémon navegando por entornos físicos para capturar, incubar y combatir a varios personajes. Su principal característica es que se debe atrapar pokémons que aparecen en diversos sitios del mundo real, como en calles, parques o algunos otros lugares de interés. Esto se consigue a través de un sistema de posicionamiento global (Global Positioning System [GPS, por sus siglas en inglés]) que indica al usuario su ubicación en tiempo real, así como la de los pokémons, pokeparadas y gimnasios pokémon, los cuales se ubican en monumentos, parques, o sitios concurridos y de referencia en el mundo real.

Los usuarios pueden elegir jugar entre ellos «combatiendo» en gimnasios pokémon, que pueden ser conquistados y utilizados para coleccionar pokécoins (la moneda virtual del juego), con la que se pueden adquirir pokebolas y otros objetos de interés dentro del juego. Cada semana Pokémon GO otorga incentivos de acuerdo a los kilómetros caminados, los cuales van desde los cinco, 25 y $50 \mathrm{~km}$. Así mismo, después de cierto tiempo aparecen pokémons con mayor dificultad para capturar, por lo que se requieren realizar «incursiones», que es una estrategia de cooperación o ayuda de otros entrenadores pokémon vinculados al juego simultáneamente en un mismo lugar (Pokemon.com, 2019).

Para cumplir el propósito de capturar pokémons, conquistar gimnasios, participar en incursiones u obtener múltiples beneficios propios del juego, el usuario debe salir a caminar, lo que implica la realización de actividad física. Estudios previos (Althoff, White \& Horvitz, 2016; EscaravajalRodríguez, 2018; Howe et al., 2016; Ma et al., 2018; Nigg, Mateo \& An, 2017; Wong, 2017) han analizado y encontrado incrementos en los niveles de actividad física de usuarios de Pokémon GO, lo que puede contribuir, o incluso alcanzar por sí mismo la cantidad de actividad física diaria recomendada (OMS, 2018).

Pokémon GO podría también incidir en el desarrollo de otras habilidades; ya que, como se ha mencionado previamente, al intentar llegar a la ubicación de los lugares de interés dentro del juego, los usuarios deben seguir un mapa virtual, el cual presenta su ubicación en tiempo real, lo que implica el uso de la capacidad de ubicación en el espacio, en este sentido, la aplicación hace uso de una brújula virtual, la cual permite determinar a los jugadores su orientación espacial; estudios como los de Escaravajal-Rodríguez (2018) y Carbonell, Saorín y Hess (2018), han encontrado que Pokémon GO puede mejorar dicha habilidad. Otro aspecto a resaltar es que, al desplazarse mientras se juega, se puede favorecer al conocimiento de espacios nuevos de la comunidad y la práctica de actividad física en medios naturales (Dorward,
Mittermeier, Sandbrook \& Spooner, 2017; EscaravajalRodríguez, 2018). También, durante las incursiones, es necesario interactuar y cooperar con otros jugadores para conseguir objetivos propuestos en el juego, lo que puede propiciar el conocimiento y socialización con nuevas personas (Escaravajal-Rodríguez, 2018; Marquet, Alberico, Adlakha \& Hipp, 2017).

Por todo lo anterior, Pokémon GO puede ser un medio que no sólo contribuya a la realización de actividad física en sus usuarios, sino también al desarrollo de habilidades sociales, conocimiento de nuevos lugares dentro de la ciudad o en el medio natural, así como mejora en la capacidad de orientación espacial. A pesar de la existencia de una amplia gama de aplicaciones para la ayuda y administración de la actividad física, también se ha encontrado que estas son más utilizadas por personas que ya cuentan con adherencia a la actividad física (Aznar et al., 2019), mientras que Pokémon GO ha logrado que un sector poblacional que tradicionalmente no realiza actividad física, la realice (Althoff et al., 2016; Escaravajal-Rodríguez, 2018; Wong, 2017), lo que incrementa la importancia de estudiar las repercusiones que la aplicación pueda tener en sus usuarios.

Se estima que en México, existen 46.7 millones usuarios de videojuegos en smartphones (Forbes.com, 2019), sin embargo, es poco lo que se conoce respecto a las repercusiones que el uso de videojuegos en teléfonos móviles pudiese tener en la actividad física, capacidad de orientación en el espacio, socialización y conocimiento de sitios nuevos en la ciudad o en el medio natural de sus usuarios, y específicamente, de Pokémon GO, por ello, el objetivo de este estudio es analizar la relación que existe entre el tiempo de actividad física realizada, la percepción de la orientación espacial, el conocimiento de sitios nuevos en la comunidad y en lugares naturales, así como la socialización de usuarios mexicanos de Pokémon GO.

\section{Método}

\section{Tipo de estudio y participantes}

El presente estudio fue de tipo descriptivo-correlacional y transversal. La muestra estuvo conformada por 911 usuarios mexicanos de Pokémon GO (706 hombres [77.5\%], 205 mujeres [22.5\%]), con edades comprendidas entre los 12 y 53 años $\left(M_{\text {edad }}=25.63, D T=6.01\right)$.

\section{Instrumento}

Se utilizó una versión adaptada al español hablado en México del cuestionario ad hoc diseñado y empleado por Escaravajal-Rodríguez (2018) el cuál consta de seis apartados en los que se plantean preguntas para conocer los antecedentes de los sujetos en la temática de Pokémon, así como actividad física, socialización, orientación y conocimiento de sitios nuevos. Además, incluye un ítem acerca de accidentes relacionados al uso del juego. La descripción del instrumento puede consultarse en el Apéndice 1.

\section{Procedimiento}

El instrumento se distribuyó de manera virtual entre los meses de junio y julio de 2019, mediante un enlace a la plataforma SurveyMonkey ${ }^{\circledR}$ que desplegaba el cuestionario, a 
través de grupos de diversas redes sociales relacionados al juego en México; 34 de ellos fueron vía Facebook, ocho pertenecientes a Twitter, cuatro de Instagram y seis a través de la app de comunicación móvil Whatsapp. En la descripción de los cuestionarios se detalló los objetivos del estudio, así como las instrucciones para su respuesta, solicitando que se realizara con honestidad y se brindó información acerca de la confidencialidad y consentimiento para el uso de los datos con fines de investigación.

\section{Análisis de datos}

Se realizaron análisis de frecuencias a los datos obtenidos y se verificó la normalidad de los mismos mediante el criterio de coeficientes de asimetría y Curtosis en rangos de -1 y +1 (Ferrando \& Anguiano-Carrasco, 2010; Muthén \& Kaplan, 1985, 1992). Además, para conocer las diferencias entre las variables estudiadas antes y después de utilizar la app de Pokémon GO, se utilizaron las pruebas t para comparar la diferencia entre las medias, así como la prueba de chicuadrado para determinar si había diferencias entre las variables con respuesta categórica.

\section{Resultados}

En la Figura 1, se presenta la distribución por regiones de México de la muestra, en ella se puede observar que la mayor parte de los participantes del estudio se encuentran distribuidos en las zonas centro $(31.8 \%)$, occidente $(26.2 \%)$ y noreste $(22 \%)$ del país.

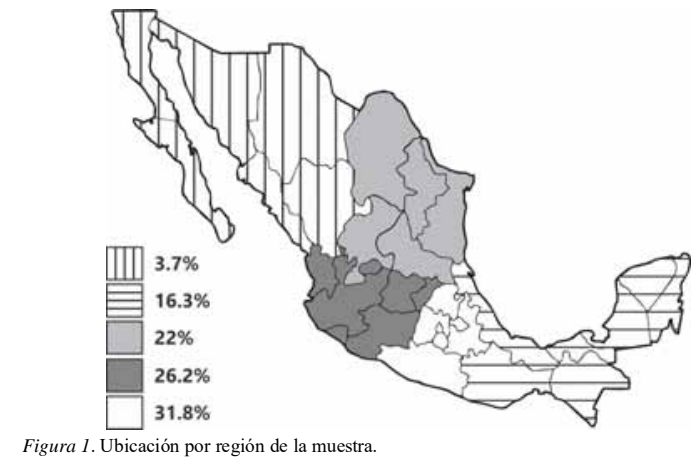

Por otra parte, con respecto a la escolaridad de los usuarios que participaron en el estudio, la mayoría mencionó contar con estudios de licenciatura (62.3\%), seguido de aquellos con bachillerato (24.4\%), con estudios de posgrado (8.9\%) y finalmente con una menor participación de personas con nivel secundaria (4.3\%).

Los datos obtenidos muestran que el $69.9 \%$ de los participantes del estudio había utilizado algún otro videojuego con la temática de Pokémon; así mismo, poco más de la mitad de los sujetos (57.5\%) indicó que lo juega desde su lanzamiento en México (en agosto de 2016); el 25\% tiene más de un año jugando y el 17.5\% lleva menos de un año.

Con relación a los días destinados a la realización de actividad física (Figura 2), se puede observar que previo a instalar la app, el 29.6\% de los sujetos no salía a caminar y sólo el $23.8 \%$ lo hacía cinco o más días por semana, mientras que después de su instalación, el $64.7 \%$ indicó que sale a caminar al menos cinco días por semana con el propósito de jugar Pokémon GO, siendo el $31.7 \%$ de la muestra, quienes salen a caminar todos los días de la semana con ese mismo objetivo. Los resultados de la prueba $t$ registraron diferencias significativas $\left(t=30.309^{(910)}, p<.001, d=2.008\right)$ entre las medias de días en que se sale a caminar antes de instalar Pokémon $\mathrm{GO}(M=2.54$ días $)$ y después de su instalación ( $M$ $=4.99$ días $)$.

Por otra parte, del total de los sujetos que reportaron no salir a caminar antes de instalar Pokémon GO (29.6\%), el 47.8\% indicó que después de hacerlo, sale a caminar al menos cinco días a la semana. Asimismo, de los sujetos que no salían a caminar antes de la instalación de Pokémon GO, la prueba $t$ de Student reveló diferencias significativas $\left(t=32.741^{(269)}, p\right.$ $<.001, d=2.821$ ) en el promedio de días que se salió a caminar después de la instalación del videojuego $(M=4.25$ días $)$.

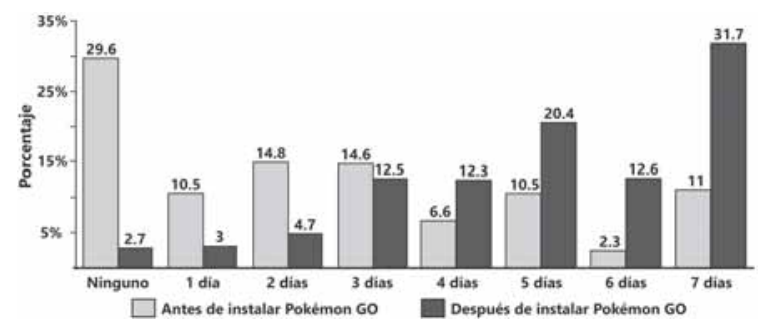

Figura 2. Frecuencia de días por semana destinados a la realización de actividad física antes y después de instalar Pokémon GO.

Respecto al tiempo destinado a caminar antes y después de jugar Pokémon GO (Figura 3), se puede apreciar que antes de instalar la app, el $76.6 \%$ de los sujetos salía a caminar menos de una hora al día, mientras que tras comenzar a jugar Pokémon GO, el 75.7\% manifestó que, con el objetivo de jugar, salen a caminar más de una hora por día. Tras realizar la prueba de chi-cuadrada $\left(\chi^{2}\right)$, se encontró que existían diferencias significativas entre la cantidad de tiempo que se destinaba a caminar antes y después de instalar Pokémon GO $\left(\chi_{(25)}^{2}=174.804, p<.001, \mathrm{~V}\right.$ de Cramer $\left.=.196\right)$.

Asimismo, del porcentaje de la muestra que expresaba no salir a caminar antes de instalar Pokémon GO $(29.6 \%$, ver Figura 2), se analizó la cantidad de tiempo al día que indicaron caminar después de la instalación de la app, encontrándose que cerca de una tercera parte de los sujetos (30.7\%) sale a caminar entre una y dos horas; poco más de una quinta parte $(21.5 \%)$, entre dos y cuatro horas; el $16.7 \%$ lo hace por más de cuatro horas; el $21.9 \%$, entre media hora y una hora; y solo $6.3 \%$, sale a caminar menos de media hora por día, esto con el propósito de jugar Pokémon GO.

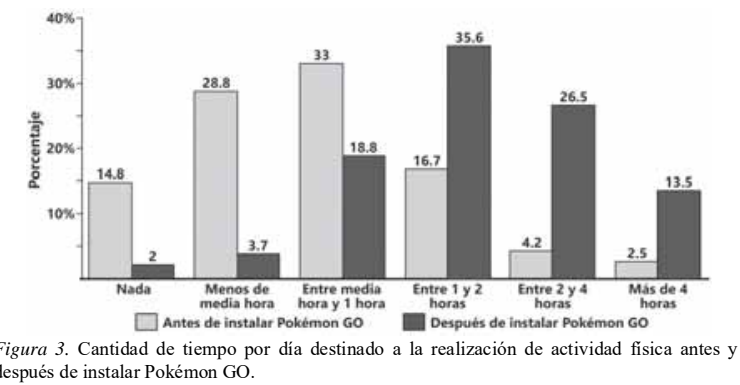

También, con referencia a actividad física realizada, el $59.4 \%$ de la muestra señaló que ha llegado a correr mientras juega Pokémon GO y el 65.1\%, dijo que el tiempo que destina a utilizar la app no ha disminuido desde que la comenzó a usar.

En cuanto a lo relacionado a la socialización, el $86.1 \%$ de 
la muestra expresó que ha conocido personas nuevas jugando Pokémon GO. En la Figura 4, se muestra que, al salir a jugar Pokémon GO, el 60.2\% de los sujetos lo hace acompañado.

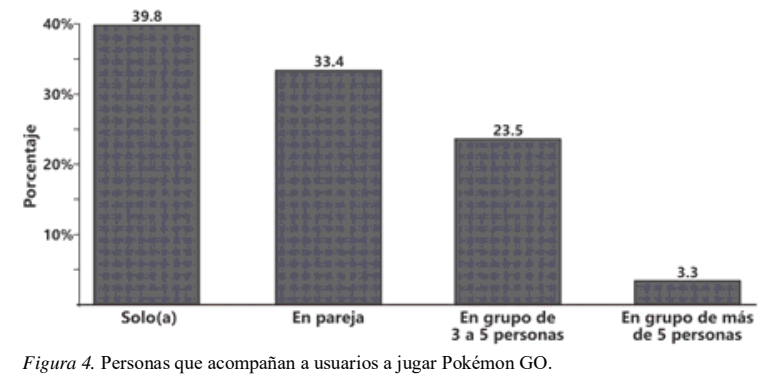

Con respecto a la capacidad de orientación espacial, la mayoría de los sujetos (84.7\%) expresaron saber que las flechas que aparecen en la parte superior derecha de la pantalla principal del juego pertenecen a los puntos cardinales norte y sur. Asimismo, la mayor parte (86.2\%) señaló nunca haberse perdido al jugar y cerca de ocho de cada 10 sujetos percibió que su capacidad de orientación utilizando un mapa virtual había mejorado jugando Pokémon GO (Figura 5).

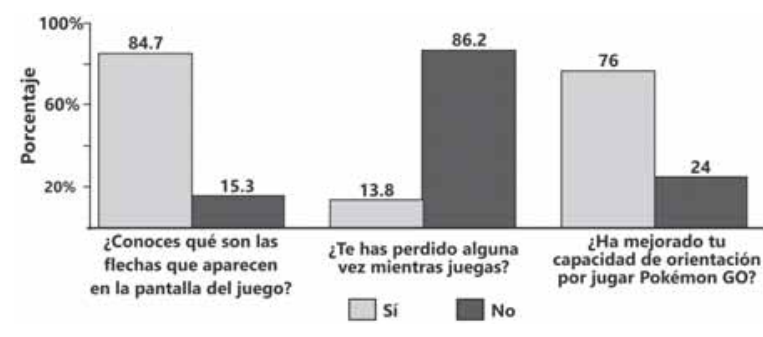

Figura 5. Aspectos relacionados a la orientación espacial al jugar Pokémon GO.

Concerniente al turismo urbano, el $89.7 \%$ de los sujetos, menciona haber conocido calles y caminos nuevos, el $88.8 \%$ monumentos y sitios nuevos de su ciudad, mientras que el $86.8 \%$, ha acudido a lugares en el medio natural.

Finalmente, la mayoría de los usuarios participantes mencionaron nunca haber sufrido accidentes al jugar Pokémon GO (70.5\%), y aquellos que sí lo tuvieron, lo sufrieron con algún elemento urbano (20.2\%) (ver Figura 6).

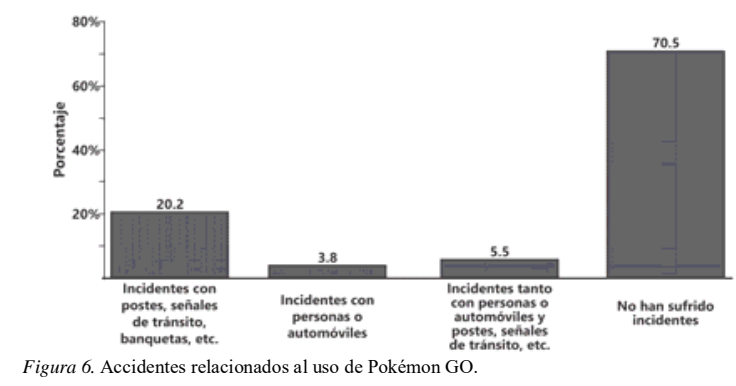

\section{Discusión}

El objetivo de este estudio fue analizar la relación que existe entre el tiempo de uso de la aplicación Pokémon GO y la cantidad de actividad física, socialización, orientación espacial, conocimiento de sitios nuevos y lugares en el medio natural, así como accidentes que se pudieran presentar al jugar por usuarios mexicanos, todo esto de manera descriptivo-correlacional y transversal.

De acuerdo a los resultados obtenidos de los participan- tes de esta investigación, se observa un incremento de la actividad física en frecuencia $\left(t=30.309^{(910)}, p<.001, d=\right.$ $2.008)$ y tiempo $\left(\chi_{(25)}^{2}=174.804, p<.001, \mathrm{~V}\right.$ de Cramer $\left.=.196\right)$ destinado a caminar posterior a la instalación de Pokémon $\mathrm{GO}$, estos datos coinciden con los reportados en usuarios españoles (Escaravajal-Rodriguez, 2018), estadounidenses (Althoff et al., 2016; Howe et al., 2016; Marquet et al., 2017) y en la región de Hong Kong (Ma et al., 2018; Wong, 2017). Por otro lado, las investigaciones realizadas en Estados Unidos y Hong Kong, encontraron que el aumento de la actividad física presentado en los usuarios de Pokémon GO disminuyó luego de un periodo de seis a cuatro semanas, contrario a este estudio, donde la mayor parte de los usuarios (65.1\%) mencionó que el tiempo que destinan al juego no ha disminuido.

En adición a lo anterior, se ha encontrado que Pokémon GO ha logrado que poblaciones que normalmente no practican actividad física, comiencen a realizarla (Althoff et al., 2016; Escaravajal-Rodríguez, 2018; Wong, 2017), en ese sentido, los resultados de este estudio encontraron que tres de cada 10 sujetos no salían a caminar ningún día antes de comenzar a jugar Pokémon GO, mientras que después de su instalación, casi seis de cada 10 personas lo hacen al menos cuatro días a la semana. De la misma manera, el 68.9\% de los sujetos reportaron caminar más de una hora por día para jugar, logrando así lo recomendado por la OMS (2018), lo que soporta la idea de que Pokémon GO ha logrado la incorporación de personas con hábitos sedentarios a la práctica de actividad física. A su vez, es importante mencionar que estos resultados deben interpretarse con cautela, debido a los sesgos que se pueden presentar a causa de la característica de autoinforme del instrumento empleado en la recolección de los datos.

Respecto a las relaciones sociales que los jugadores de Pokémon GO podrían establecer, los resultados muestran que un porcentaje importante $(86.1 \%)$ de los sujetos reportó haber conocido gente nueva al jugar, mientras que seis de cada 10 personas manifestaron que salen a jugar acompañados. Esto puede indicar que el uso de Pokémon GO, podría estar relacionado a mejoras en la socialización de los jugadores, aunque esta relación debe ser estudiada con mayor profundidad. Los resultados obtenidos van acorde a lo encontrado por Escaravajal-Rodríguez (2018) y Marquet et al. (2017) quienes exponen que Pokémon GO mejora las relaciones sociales y comunicación entre sus usuarios.

Autores como Escaravajal-Rodríguez (2018) y Carbonell et al. (2018), señalan que el uso de Pokémon GO podría asociarse a la mejora de habilidades de orientación espacial, y los resultados de este estudio apoyan esa idea, ya que los participantes manifestaron conocer el significado de la brújula que aparece en la pantalla principal del juego, no haberse perdido nunca mientras jugaban y que su capacidad de orientación utilizando un mapa virtual, había mejorado jugando Pokémon GO, sin embargo, es necesario realizar estudios bajo una metodología experimental que permitan dar mayor sustento a esta asociación.

También se identificó que el uso de Pokémon GO favorece el conocimiento de nuevos sitios, monumentos y calles, lo que permite tener nuevas experiencias turísticas de espacios urbanos y la interacción en el medio natural, teniendo 
concordancia con lo señalado en estudios con otras poblaciones (Escaravajal-Rodríguez, 2018; Dorward et al., 2017; Ma et al., 2018).

Además, jugar Pokémon GO se ha relacionado a algunos accidentes debido a la distracción que puede provocar mientras se juega (Wagner-Greene et al., 2017), por ello, se hace indispensable tomar las debidas precauciones para evitarlos, ya que en este estudio se presentó una prevalencia del $30 \%$.

De la misma manera, es importante mencionar que este estudio contó con algunas limitaciones, en primer lugar, en la medición de la cantidad de actividad física realizada, socialización, orientación espacial y conocimiento de sitios nuevos y visita de áreas en el medio natural, como se mencionó con anterioridad, se empleó un instrumento con características de autoinforme, por lo que podría existir un sesgo en las respuestas proporcionadas debido a imprecisiones en los recuerdos de los usuarios que participaron en el estudio. Por otra parte, debido a la manera en que se distribuyó el cuestionario, no se consiguió una muestra proporcional entre hombres y mujeres, así como representativa de usuarios mexicanos de Pokémon GO. Tomando en cuenta lo anterior, futuros estudios podrían medir de manera más objetiva la cantidad de actividad física realizada (mediante instrumentos como GPS, pulsómetros, acelerómetros o podómetros para relacionar el gasto energético a partir de la actividad física) al jugar Pokémon GO, así como la mejora en la socialización, orientación en el espacio e incremento en la frecuencia y tiempo de visita a espacios en el medio natural. Además, sería un gran aporte tomar en cuenta el monitoreo de marcadores biológicos (e.g., glucosa, colesterol, triglicéridos, entre otros) relacionados a problemas de salud ocasionados por el sedentarismo, para poder determinar si el uso de la app, incide en ellos. Finalmente, se podrían realizar comparaciones con otras aplicaciones móviles de características similares (e.g., Ingress, Harry Potter: Wizards Unite o Jurassic World Alive), que permitan conocer aspectos que llamen la atención de los usuarios para su utilización.

\section{Conclusiones}

Este estudio ha permitido identificar un incremento de la actividad física y una mejor percepción de habilidades de orientación en el espacio a partir del uso de la aplicación de Pokémon GO, sus usuarios, generalmente lo hacen acompañados, lo que les permite incrementar su interacción, el conocimiento de nuevos lugares para un turismo urbano y en el medio natural. Pese a ello, durante su uso no se debe dejar de prestar atención a lo que sucede en el entorno, ya que existe una probabilidad importante de accidentes con elementos urbanos.

\section{Contribuciones}

Hasta el momento de la redacción de este manuscrito, no se han reportado estudios donde se analice la relación de Pokémon GO con la actividad física realizada en usuarios latinoamericanos, por lo que los resultados presentados, representan una contribución inicial importante que permita comprender los efectos que Pokémon GO, e incluso otras aplicaciones gamificadas que tengan como elemento inherente al juego la realización de actividad física, puedan tener sobre sus usuarios.

\section{Referencias}

Aguilar-Martínez, A., Tort, E., Medina, F. X., \& Saigí-Rubió, F. (2015). Posibilidades de las aplicaciones móviles para el abordaje de la obesidad según los profesionales. Gaceta Sanitaria,29(6), 419-424. doi:10.1016/j.gaceta.2015.07.014

Althoff, T., White, R. W., \& Horvitz, E. (2016). Influence of Pokémon Go on physical activity: study and implications. Journal of medical Internet research, 18(12), e315. doi: 10.2196/jmir.6759

Ayenigbara, I. O. (2018). Gaming Disorder and Effects of Gaming on Health: An Overview. Journal of Addiction Medicine and Therapeutic Science, 4(1), 1-3. doi: 10.17352/24553484.000025

Aznar, I., Cáceres, M., Trujillo, J., \& Romero, J. (2019). Impacto de las apps móviles en la actividad física: un meta-análisis. Retos, 36(36), 52-57.

Buckley, K. E., \& Anderson, C. A. (2006). A theoretical model of the effects and consequences of playing video games. En P. Vorderer \& J. Bryant (Eds.), Playing Video Games - Motives, Responses, and Consequences (pp. 363-378). Mahwah, NJ: LEA.

Carbonell, C., Saorín, J. L., \& Hess, S. (2018). Pokémon GO and improvement in spatial orientation skills. Journal of Geography, 117(6), 245-253. doi:10.1080/ 00221341.2018 .1470663

Daley, A. J. (2009). Can Exergaming Contribute to Improving Physical Activity Levels and Health Outcomes in Children? Pediatrics, 124(2), 763-771. doi: 10.1542/peds.2008-2357

Dorward, L. J., Mittermeier, J. C., Sandbrook, C., \& Spooner, F. B. (2017). Pokémon Go: Benefits, Costs, and Lessons for the Conservation Movement: Conservation implications of Pokémon Go. Conservation Letters, 10(1), 160-165. doi: 10.1111/conl.12326

Escaravajal-Rodríguez, J. C. (2018). Pokémon GOy su influencia en usuarios españoles de Facebook. Apunts. Educación Física y Deportes. Educación física y deportes, 3(133), 3849. doi: 10.5672/apunts.2014-0983.es.(2018/3).133.03

Ferrando,P.J., \&Anguiano-Carrasco, C.(2010). El análisis factorial como técnica de investigación en psicología. Papeles del Psicólogo, 31(1), 18-33.

Ferguson, C. J. (2007). The Good, The Bad and the Ugly: AMetaanalytic Review of Positive and Negative Effects of Violent Video Games. Psychiatric Quarterly, 78(4), 309-316. doi: 10.1007/s11126-007-9056-9

Forbes.com (2019). Análisis | En cuestión de videojuegos, \#JugarYaNoEsCosadeNiños. Recuperado de: http:// test.forbes.com.mx/analisis-en-cuestion-de-videojuegosjugaryanoescosadeninos/

Greitemeyer, T., \& Mügge, D. O. (2014). Video Games DoAffect Social Outcomes: A Meta-Analytic Review of the Effects of Violent and Prosocial Video Game Play. Personality and Social Psychology Bulletin, 40(5), 578-589. doi: 10.1177/ 0146167213520459

Hallal, P. C., Wells, J. C., Reichert, F. F., Anselmi,L., \& Victora, C. G (2006). Early determinants of physical activity in adolescence: prospective birth cohort study. BMJ, 332, 1002-1007. 
doi:10.1136/bmj.38776.434560.7C

Howe, K. B., Suharlim, C., Ueda, P., Howe, D., Kawachi, I., \& Rimm, E. B. (2016). Gotta catch'em all! Pokémon GO and physical activity among young adults: difference in differences study. BMJ, 355, i6270. doi: 10.1136/bmj.i6270

Instituto Nacional de Estadística y Geografía. (2018). Encuesta Nacional sobre Disponibilidad y Uso de TIC en Hogares (ENDUTIH). Recuperado de https://datos.gob.mx/busca/ dataset/encuesta-nacional-sobre-disponibilidad-y-uso-detic-en-hogares-endutih

Khang, H., Kim, J. K., \& Kim, Y. (2013). Self-traits and motivations as antecedents of digital media flow and addiction: The Internet, mobile phones, and video games. Computers in Human Behavior, 29(6), 2416-2424. doi:10.1016/ j.chb.2013.05.027

Klitsie, T., Corder, K., Visscher, T. L. S.,Atkin,A. J., Jones, A. P., \& van Sluijs, E. M. F. (2013). Children's sedentary behaviour: descriptive epidemiology and associations with objectivelymeasured sedentary time. BMC Public Health, 13, 1092. doi: 10.1186/1471-2458-13-1092

López, J., Pozo, S., Fuentes, A., \& Vicente, M. R. (2019). Los juegos populares como recurso didáctico para la mejora de hábitos de vida saludables en la era digital. Retos, 36, 266272.

López, S., Suárez, S., Ruiz, A., \& Martínez, E. J.(2017).Exergames: videojuegos activos para la promoción de la actividad física y el desarrollo cognitivo en educación primaria y secundaria. Sevilla, España: Wanceulen.

Lozano-Sánchez, A. M., Zurita-Ortega, F., Ubago-Jiménez, J. L., Puertas-Molero, P., Ramírez-Granizo, I., \& Núñez-Quiroga, J. I. (2019). Videojuegos, práctica de actividad física, obesidad y hábitos sedentarios en escolares de entre 10 y 12 años de la provincia de Granada. Retos, 35, 42-46.

Lunde, P., Nilsson, B. B., Bergland, A., Kværner, K. J., \& Bye, A. (2018). The effectiveness of smartphone apps for lifestyle improvement in noncommunicable diseases: systematic review and meta-analyses. Journal of medical Internet research, 20(5), e162. doi: 10.2196/jmir.9751

Ma, B. D., Ng, S. L., Schwanen, T., Zacharias, J., Zhou, M., Kawachi, I., \& Sun, G. (2018). Pokémon GO and physical activity in Asia: multilevel study. Journal of medical Internet research, 20(6), e217. doi: 10.2196/jmir.9670

Marquet, O., Alberico, C., Adlakha, D., \& Hipp, J. A. (2017). Examining Motivations to Play Pokémon GO and Their Influence on Perceived Outcomes and Physical Activity. JMIR Serious Games, 5(4), e21. doi: 10.2196/games.8048

Moncada, J., \& Chacón, Y. (2012). El efecto de los videojuegos en variables sociales, psicológicas y fisiológicas en niños y adolescentes. Retos, 21(21), 43-49.

Muthén, B., \& Kaplan D. (1985). A comparison of some methodologies for the factor analysis of non-normal Likert variables. British Journal of Mathematical and Statistical Psychology, 38(2), 171-189. doi:10.1111/j.20448317.1985.tb00832.x

Muthén, B., \& Kaplan D. (1992). A comparison of some methodologies for the factor analysis of non-normal Likert variables: A note on the size of the model. British Journal of Mathematical and Statistical Psychology, 45(1), 19-30. doi:10.1111/j.2044-8317.1992.tb00975.x

Nianticlibs.com. (2019). Los juegos redefinen nuestra realidad. Recuperado de https://nianticlabs.com/es/products/

Nigg, C. R., Mateo, D. J., \& An, J. (2017). Pokémon GO may increase physical activity and decrease sedentary behaviors.
American journal of public health, 107(1),37-38. doi: 10.2105/ AJPH.2016.303532

Organización Mundial de la Salud. (2018). Actividad física. Recuperado de https://www.who.int/es/news-room/factsheets/detail/physical-activity

Panova, T., \& Carbonell, X. (2018). Is smartphone addiction really an addiction?. Journal of Behavioral Addictions, 7(2), 252259. doi: 10.1556/2006.7.2018.49

Peng, W., Crouse, J. C., \& Lin, J. (2012). Using Active Video Games for Physical Activity Promotion: A Systematic Review of the Current State of Research. Health Education \& Behavior, 40(2), 171-192. doi: 10.1177/1090198112444956

Pokemon.com. (2019). Pokémon GO. Recuperado de https:// www.pokemon.com/es/app/pokemon-go/

Staiano, A. E., \& Calvert, S. L. (2011). Exergames for Physical Education Courses: Physical, Social, and Cognitive Benefits. Child Development Perspectives, 5(2), 93-98. doi:10.1111/ j.1750-8606.2011.00162.x

Sween, J., Wallington, S. F., Sheppard, V., Taylor, T., Llanos, A.A., \&Adams-Campbell, L. L. (2014). The Role of Exergaming in Improving Physical Activity: A Review. Journal of Physical Activity and Health, 11(4), 864-870. doi: 10.1123/jpah.20110425

Wagner-Greene, V. R., Wotring, A. J., Castor, T., Kruger, J., Mortemore, S., \& Dake, J.A. (2017). Pokémon GO: Healthy or harmful?. American journal of public health, 107(1), 35-36.

Wang, D., Xiang, Z., \& Fesenmaier, D. R. (2016). Smartphone use in everyday life and travel. Journal of travel research, 55(1), 52-63. doi:10.1177/0047287514535847

Wong, F. Y. (2017). Influence of Pokémon Go on physical activity levels of university players: a cross-sectional study. International journal of health geographics, 16(8), 1-12. doi: 10.1186/s12942-017-0080-1

Apéndice 1. Adaptación al español hablado en México del Cuestionario hábitos y prácticas de Pokémon GO relacionadas a la actividad física (Escaravajal-Rodríguez, 2018). Antecedentes

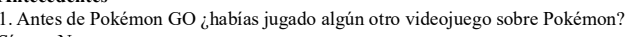

Sí_ No

2. ¿Hace cuánto que juegas Pokémon GO?

Desde que se lanzó__ Menos de 1 año__ Más de 1 año__ Más de 2 años_

Prácticas y hábitos relacionados con la actividad física

3. Antes de que instalaras la app de Pokémon $\mathrm{GO}_{¿}$ Cuántos días a la semana salías a caminar (para jugar Pokémon GO)?

Nunca_ 1 día_ 2 días_ 3 días_ 4 días_ 5 días_ 6 días_ 7 días

4. Después de que instalaras la app de Pokémon GO ¿Cuántos días a la semana sales a caminar (para jugar Pokémon GO)?

Nunca_ 1 día_2 días 3 días_ 4 días_ 5 días_ 6 días 7 días

5. Antes de que instalaras la app de Pokémon GO ¿Cuánto tiempo al día salías a caminar (para jugar Pokémon GO)?

Nada__ Menos de una hora__Entre 1 y 2 horas_Entre 2 y 4 horas__ Más de 4 horas

6. Después de que instalaras la app de Pokémon $\overline{\mathrm{GO}}$ ¿Cuánto tiempo al día sales a caminar (para ugar Pokémon GO)

Nada__ Menos de una hora_Entre 1 y 2 horas__Entre 2 y 4 horas__ Más de 4 horas

7. Después de que instalaras la app de Pokémon $\overline{\mathrm{GO}}$ ¿Ha disminuido el tiempo que destinas a ella? Sí_No

8. ¿Has llegado a correr mientras juegas Pokémon GO?

Sí No

Socialización

9. Cuando sales a jugar Pokémon GO ¿normalmente lo haces sólo(a) o acompañado(a)?

Sólo(a)__En pareja__ En grupo de 3 a 5 personas___ En grupos de más de 5 personas_

10. Jugando Pokémon $\overline{\mathrm{GO}}$ ¿Has conocido nuevas personas?

Sí_ No

Habilidades de orientación

11. En la pantalla principal del juego y arriba a la derecha, hay una flecha con un lado rojo y otro gris ¿Sabías que representan el norte y sur?

Sí_ No

12. ¿Te has perdido alguna vez mientras jugabas Pokémon GO?

Sí No

13. ¿Crees que tu capacidad de orientación con mapa virtual ha mejorado debido a jugar Pokémon $\mathrm{GO}$ ?

Sí_ No

Conocimiento de sitios nuevos

14. Al jugar Pokémon GO ihas ido por sitios y/o calles nuevas por los que nunca habías pasado?

Sí No

Sí No No

15. Al jugar

Sí_No - No ido a espacios naturales como parques, playa, montaña bosques o aguno similar

16. ¿Has ido a espacios
busca de un pokémon?

Sí_ No

Aí No No

17. ¿Has tenido algún percance/accidente mientras jugabas Pokémon GO?

Sí, me he tropezado con algún elemento urbano (poste, señal de tránsito, escalón, banqueta, etc.)

Sí, me he tropezado con alghin elemento urb

Sí, he chocado con alguna persona o coche
Sí, me he tropezado/chocado tanto con algún elemento urbano como con alguna persona o coche No

Retos, número 38, 2020 ( $2^{\circ}$ semestre) 